\title{
A modified proximal point algorithm for solving variational inclusion problem in real Hilbert spaces
}

\author{
Thierno M. M. Sow \\ Communicated by Hongyu Liu
}

\begin{abstract}
The purpose of this paper is to use a modified proximal point algorithm for solving variational inclusion problem in real Hilbert spaces. It is proven that the sequence generated by the proposed iterative algorithm converges strongly to the common solution of the convex minimization and variational inclusion problems.
\end{abstract}

Keywords. Proximal point algorithms, convex minimization problem, variational inclusion problem, forward-backward splitting method.

2010 Mathematics Subject Classification. 47H05, 47H09, 47J25.

\section{Introduction}

Let $H$ be a real Hilbert space and $K$ be a nonempty, closed, and convex subset of $H$. Consider the following convex minimization problem:

$$
\begin{aligned}
& \text { Minimize } f(x) \\
& \text { subject to } x \in K \text {, }
\end{aligned}
$$

where $f: K \rightarrow \mathbb{R}$ is a real-valued convex function. The set of all minimizers of $g$ on $H$ is denoted by $\operatorname{argmin}_{y \in K} f(y)$. A successful and powerful tool for solving problem (1) is the well-known proximal point algorithm (shortly, the PPA) which was initiated by Martinet [14] in 1970 and later studied by Rockafellar [18] in 1976. Let $H$ be a real Hilbert space. The PPA is defined as follows:

$$
\left\{\begin{array}{l}
x_{1} \in H, \\
x_{n+1}=\operatorname{argmin}_{y \in H}\left[f(y)+\frac{1}{2 \lambda_{n}}\left\|x_{n}-y\right\|^{2}\right],
\end{array}\right.
$$

where $\lambda_{n}>0$ for all $n \geq 1$. In [18] Rockafellar proved that the sequence $\left\{x_{n}\right\}$ given by (2) converges weakly to a minimizer of $f$. In the recent years, the problem of finding a solution of problem (1) by using a modified proximal point algorithm 
in real Hilbert spaces has been intensively studied by many authors; see, for example, $[8,10,11,19,21]$ and the references therein.

Let $A: H \rightarrow H$ be a single-valued nonlinear mapping and $B: H \rightarrow 2^{H}$ be a set-valued mapping. The variational inclusion problem is as follows:

Find $x \in H$ such that

$$
0 \in B(x)+A(x) .
$$

We denote the set of solutions of this problem by $(A+B)^{-1}(0)$. If $A=0$, then problem (3) becomes the inclusion problem introduced by Rockafellar [18]. Inclusions of the form specified by (3) arise in numerous problems of fundamental importance in mathematical optimization, either directly or through an appropriate reformulation. In general, equations of inclusion monotone type (3) are nonlinear and there is no known method to find closed form solutions for them. Consequently, methods of approximating solutions of such equations are of interest. A popular method for solving problem (3) is the well-known forward-backward splitting method introduced by Passty [16] and Lions and Mercier [12].The method is formulated as

$$
x_{n+1}=\left(I-\lambda_{n} B\right)^{-1}\left(I-\lambda_{n} A\right) x_{n}, \lambda_{n}>0,
$$

under the condition that $D(B) \subset D(A)$. It was shown, see for example [4], that weak convergence of (4) requires quite restrictive assumptions on $A$ and $B$, such that the inverse of $A$ is strongly monotone or $B$ is Lipschitz continuous and monotone and the operator $A+B$ is strongly monotone on $D(B)$. Hence, the modification is necessary in order to guarantee the strong convergence of forward-backward splitting method (see, for example, [7, 9, 22-24] and the references contained in them). In recent years, monotone operators have received a lot of attention for treating zero points of monotone operators; see [4,8-12,16,24] and the references therein. Very recently, Boikanyo [2] used proximal point algorithm for finding zero points of the sum of two operators such that the sequence of error terms is square summable in norm. He proved the following theorem.

Theorem 1.1. Let $H$ be a real Hilbert space and let $f: H \rightarrow H$ be $a k$ contraction with $k<\frac{1}{2}$. Assume that $A: D(A)=H \rightarrow H$ is a $\beta$-inverse strongly operator and $B: D(B) \subset H \rightarrow H$ is a maximal monotone operator with $S:=(A+B)^{-1}(0) \neq \emptyset$. For $x_{0} \in H$, let $\left(x_{n}\right)$ be a sequence generated by

$$
\left\{\begin{array}{l}
x_{0} \in H, \\
x_{n+1}=\alpha_{n} f\left(x_{n}\right)+\gamma_{n} x_{n}+\delta_{n} J_{r_{n}}^{B}\left(x_{n}-r_{n} A x_{n}\right)
\end{array}\right.
$$


with $\alpha_{n} \in(0,1), \gamma_{n} \in\left(-\frac{3}{2}, 1\right)$, and $\delta_{n} \in\left(0, \frac{3}{2}\right)$ satisfying $\alpha_{n}+\gamma_{n}+\delta_{n}=1$, $r_{n} \in(0, \beta]$, and $\left(e_{n}\right)$ is a sequence of errors in $H$. Then, $\left(x_{n}\right)$ converges strongly to the unique fixed point $z$ of $P_{S} f$, provided that

(i) $\lim _{n \rightarrow \infty} \alpha_{n}=0$ and $\sum_{n=0}^{\infty} \alpha_{n}=\infty$.

(ii) $\liminf r_{n}>0$,

(iii) $0<\liminf \delta_{n}<\limsup \delta_{n}<\frac{3}{2}$,

(iv) $\left\|e_{n}\right\| \leq \gamma_{n}\left\|x_{n}-\delta_{n} J_{r_{n}}^{B}\left(x_{n}-r_{n} A x_{n}\right)\right\|$ with $\sum_{n=0}^{\infty} \gamma_{n}^{2}<\infty$.

Motivated by Boikanyo [2] and Rockafellar [18], we construct an iterative algorithm and strong convergence theorems for finding a point in the intersection of the set of solution of variational inclusion problem and the set of solution of convex minimization problem is established. Finally, our method of proof is of independent interest.

\section{Preliminaries}

In this section, we give some preliminaries, definitions and results which will be needed in the sequel.

Let $A: K \rightarrow H$ be a single-valued nonlinear mapping and $A$ is $\alpha$-inverse strongly monotone if there exists a constant $\alpha>0$ such that

$$
\langle A x-A y, x-y\rangle \geq \alpha\|A x-A y\|^{2}, \forall x, y \in K .
$$

It is immediate that if $A$ is $\alpha$-inverse strongly monotone, then $A$ is monotone and Lipschitz continuous.

The demiclosedness of a nonlinear operator $T$ usually plays an important role in dealing with the convergence of fixed point iterative algorithms.

Lemma 2.1. [3] Let $H$ be a real Hilbert space $H$ and $T: H \rightarrow H$ be a nonexpansive mapping such that $\operatorname{Fix}(T) \neq \emptyset$. Then $I-T$ is demiclosed; that is,

$$
\left\{x_{n}\right\} \subset H, x_{n} \rightarrow x \in K \text { and }(I-T) x_{n} \rightarrow y \text { implies that }(I-T) x=y .
$$

Lemma 2.2. [20] Let $H$ be a real Hilbert space and $A: H \rightarrow H$ be an $\alpha$ inverse strongly monotone mapping. Then, $I-\theta A$ is nonexpansive mapping for all $x, y \in H$ and $\theta \in[0,2 \alpha]$. 
Lemma 2.3 (Chidume, [5]). Let $H$ be a real Hilbert space. Then, for every $x, y \in$ $H$, and every $\lambda \in[0,1]$, the following holds:

$$
\|x+y\|^{2} \leq\|x\|^{2}+2\langle y, x+y\rangle \text {. }
$$

Lemma $2.4(\mathrm{Xu},[23])$. Assume that $\left\{\beta_{n}\right\}$ is a sequence of nonnegative real numbers such that $\beta_{n+1} \leq\left(1-\varepsilon_{n}\right) \beta_{n}+\gamma_{n}$ for all $n \geq 0$, where $\left\{\varepsilon_{n}\right\}$ is a sequence in $(0,1)$ and $\left\{\gamma_{n}\right\}$ is a sequence in $\mathbb{R}$ such that
(a) $\sum_{n=0}^{\infty} \varepsilon_{n}=\infty$,
(b) $\limsup _{n \rightarrow \infty} \frac{\gamma_{n}}{\varepsilon_{n}} \leq 0$ or $\sum_{n=0}^{\infty}\left|\gamma_{n}\right|<\infty$. Then $\lim _{n \rightarrow \infty} \beta_{n}=0$.

For every $\lambda>0$, the Moreau-Yosida resolvent of $f, J_{\lambda}^{f}$ is defined by :

$$
J_{\lambda}^{f} x=\operatorname{argmin}_{u \in H}\left[f(u)+\frac{1}{2 \lambda}\|x-u\|^{2}\right],
$$

for all $x \in H$. It was shown in [8] that the set of fixed points of the resolvent associated to $F$ coincides with the set of minimizers of $f$. Also, the resolvent $J_{\lambda}^{f}$ of $f$ is nonexpansive for all $\lambda>0$.

Lemma 2.5. (Miyadera [15]) Let $f: K \rightarrow(-\infty,+\infty)$ be a proper, lower semicontinuous and convex function. For every $r>0$ and $\mu>0$, the following holds:

$$
J_{r}^{f} x=J_{\mu}^{f}\left(\frac{\mu}{r} x+\left(1-\frac{\mu}{r}\right) J_{r}^{f} x\right) .
$$

Lemma 2.6 (Sub-differential inequality, Ambrosio et al. [1]). Let $f: H \rightarrow$ $(-\infty,+\infty)$ be a proper, lower semicontinuous and convex function. Then, for every $x, y \in H$ and $\lambda>0$, the following sub-differential inequality holds:

$$
\frac{1}{\lambda}\left\|J_{\lambda}^{f} x-y\right\|^{2}-\frac{1}{\lambda}\|x-y\|^{2}+\frac{1}{\lambda}\left\|x-J_{\lambda}^{f} x\right\|^{2}+f\left(J_{\lambda}^{f} x\right) \leq f(y) .
$$

Let a set-valued mapping $B: H \rightarrow 2^{H}$ be a maximal monotone. We define a resolvent operator $J_{\lambda}^{B}$ generated by $B$ and $\lambda$ as follows:

$$
J_{\lambda}^{B}=(I+\lambda B)^{-1}(x) \forall x \in H,
$$

where $\lambda$ is a positive number. It is easily to see that the resolvent operator $J_{\lambda}^{B}$ is single-valued, nonexpansive and 1-inverse strongly monotone and moreover, a solution of problem (3) is a fixed point of the operator $J_{\lambda}^{B}(I-\lambda A)$ for all $\lambda>0$ (see, for example, [6]).

Lemma 2.7. [12] Let $B: H \rightarrow 2^{H}$ be a maximal monotone mapping and $A$ : $H \rightarrow H$ be a Lipschitz and continuous monotone mapping. Then, the mapping $B+A: H \rightarrow 2^{H}$ is maximal monotone. 


\section{Main Result}

We are in a position to state and investigate the new proximal iterative method for finding a common element of the set of minimizers of a convex function and the set of solutions of variational inclusion with set-valued maximal monotone mapping and inverse strongly monotone mapping in real Hilbert spaces.

Theorem 3.1. Let $K$ be a nonempty closed convex subset of a real Hilbert space H. Let $g: K \rightarrow(-\infty,+\infty]$ be a proper, lower semi-continuous and convex function and $A$ be an $\alpha$-inverse strongly monotone operator of $K$ into $H$. Let $f: K \rightarrow K$ be a b-contraction mapping and $B$ be a maximal monotone operator on $H$ such that $\Gamma:=\operatorname{argmin}_{u \in K} g(u) \cap(A+B)^{-1}(0)$ is non-empty and the domain of $B$ is included in K. Let $\left\{x_{n}\right\}$ be a sequence defined as follows:

$$
\left\{\begin{array}{l}
x_{0} \in K, \\
u_{n}=\operatorname{argmin}_{u \in K}\left[g(u)+\frac{1}{2 \lambda_{n}}\left\|u-x_{n}\right\|^{2}\right], \\
x_{n+1}=\alpha_{n} f\left(x_{n}\right)+\left(1-\alpha_{n}\right) J_{\theta_{n}}^{B}\left(u_{n}-\theta_{n} A u_{n}\right),
\end{array}\right.
$$

where $\left\{\alpha_{n}\right\},\left\{\lambda_{n}\right\}$, and $\left\{\theta_{n}\right\}$ be sequences in $(0,1)$ and $\lambda_{n} \geq \lambda>0$ for all $n \geq 1$ and some $\lambda$ satisfying the following conditions:

(i) $\lim _{n \rightarrow \infty} \alpha_{n}=0$ and $\sum_{n=0}^{\infty} \alpha_{n}=\infty$,

(ii) $\theta_{n} \in[a, b] \subset(0, \min \{1,2 \alpha\})$.

Then, the sequence $\left\{x_{n}\right\}$ generated by (7) converges strongly to $p \in \Gamma$, which is the unique solution of the variational inequality problem:

$$
\langle p-f(p), p-q\rangle \leq 0, \quad \forall q \in \Gamma .
$$

Proof. By using property of $(I-f)$ and since $\Gamma$ is closed convex, the variational inequality (8) has a unique solution in $\Gamma$. We denote by $p$ the unique solution of (8). Using (7) and properties of $g$, we have $g(p) \leq g(u)$ for all $u \in K$. This implies that

$$
g(p)+\frac{1}{2 \lambda_{n}}\|p-p\|^{2} \leq g(u)+\frac{1}{2 \lambda_{n}}\|u-p\|^{2}
$$

and hence $J_{\lambda_{n}}^{g} p=p$ for all $n \geq 1$, where $J_{\lambda_{n}}^{g}$ is the Moreau-Yosida resolvent of $g$ in $K$. Therefore,

$$
\left\|u_{n}-p\right\|=\left\|J_{\lambda_{n}}^{g} x_{n}-p\right\| \leq\left\|x_{n}-p\right\|
$$


From (7), the fact that $p=J_{\theta_{n}}^{B}\left(I-\theta_{n} A\right) p$ and Lemma 2.2, we have

$$
\left\|J_{\theta_{n}}^{B}\left(I-\theta_{n} A\right) u_{n}-p\right\| \leq\left\|u_{n}-p\right\| \leq\left\|x_{n}-p\right\|, \quad \forall n \geq 0 .
$$

Hence,

$$
\begin{aligned}
\left\|x_{n+1}-p\right\| \leq & \left\|\alpha_{n} f\left(x_{n}\right)+\left(1-\alpha_{n}\right) J_{\theta_{n}}^{B}\left(I-\theta_{n} A\right) u_{n}-p\right\| \\
\leq & \alpha_{n}\left\|f\left(x_{n}\right)-f(p)\right\|+\left(1-\alpha_{n}\right) \| J_{\theta_{n}}^{B}\left(I-\theta_{n} A\right) u_{n} \\
& -p\left\|+\alpha_{n}\right\| f(p)-p \| \\
\leq & \left(1-\alpha_{n}(1-b)\right)\left\|x_{n}-p\right\|+\alpha_{n}\|f(p)-p\| \\
\leq & \max \left\{\left\|x_{n}-p\right\|, \frac{\|f(p)-p\|}{1-b}\right\} .
\end{aligned}
$$

By induction on $n$, we obtain that

$$
\left\|x_{n}-p\right\| \leq \max \left\{\left\|x_{0}-p\right\|, \frac{\|f(p)-p\|}{1-b}\right\}, \quad n \geq 1 .
$$

Hence $\left\{x_{n}\right\}$ is bounded. Now, we prove that $x_{n} \rightarrow p$.

We divide the proof into two cases.

Case 1. Assume that there is $n_{0} \in N$ such that $\left\{\left\|x_{n}-x^{*}\right\|\right\}$ is decreasing for all $n \geq n_{0}$. Since $\left\{\left\|x_{n}-x^{*}\right\|\right\}$ is monotonic and bounded, $\left\{\left\|x_{n}-x^{*}\right\|\right\}$ is convergent. Clearly, we have

$$
\lim _{n \rightarrow \infty}\left(\left\|x_{n}-p\right\|^{2}-\left\|x_{n+1}-p\right\|^{2}\right)=0 .
$$

By using Lemma 2.6 and since $g(p) \leq g\left(u_{n}\right)$, we get

$$
\left\|x_{n}-u_{n}\right\|^{2} \leq\left\|x_{n}-p\right\|^{2}-\left\|u_{n}-p\right\|^{2} .
$$

Therefore, from (7), inequality (11) and convexity of $\|\cdot\|^{2}$, we get that

$$
\begin{aligned}
\left\|x_{n+1}-p\right\|^{2} & =\left\|\alpha_{n} f\left(x_{n}\right)+\left(1-\alpha_{n}\right) J_{\theta_{n}}^{B}\left(I-\theta_{n} A\right) u_{n}-p\right\|^{2} \\
& \leq\left(1-\alpha_{n}\right)\left\|J_{\theta_{n}}^{B}\left(I-\theta_{n} A\right) u_{n}-p\right\|^{2}+\alpha_{n}\left\|f\left(x_{n}\right)-p\right\|^{2} \\
& \leq\left(1-\alpha_{n}\right)\left\|u_{n}-p\right\|^{2}+\alpha_{n}\left\|f\left(x_{n}\right)-p\right\|^{2} \\
& \leq\left(1-\alpha_{n}\right)\left(\left\|x_{n}-p\right\|^{2}-\left\|x_{n}-u_{n}\right\|^{2}\right)+\alpha_{n}\left\|f\left(x_{n}\right)-p\right\|^{2} .
\end{aligned}
$$

Hence,

$$
\left(1-\alpha_{n}\right)\left\|x_{n}-u_{n}\right\|^{2} \leq\left\|x_{n}-p\right\|^{2}-\left\|x_{n+1}-p\right\|^{2}+\alpha_{n}\left\|f\left(x_{n}\right)-p\right\|^{2} .
$$


It then implies from (10) and $\alpha_{n} \rightarrow 0$ that

$$
\lim _{n \rightarrow \infty}\left\|x_{n}-u_{n}\right\|^{2}=0
$$

From (7) and Lemma 2.2, it follows that

$$
\begin{gathered}
\left\|x_{n+1}-p\right\|^{2}=\left\|\alpha_{n}\left(f\left(x_{n}\right)-p\right)+\left(1-\alpha_{n}\right)\left(J_{\theta_{n}}^{B}\left(I-\theta_{n} A\right) u_{n}-p\right)\right\|^{2} \\
\leq \alpha_{n}\left\|f\left(x_{n}\right)-p\right\|^{2}+\left(1-\alpha_{n}\right)\left\|J_{\theta_{n}}^{B}\left(I-\theta_{n} A\right) u_{n}-p\right\|^{2} \\
=\alpha_{n}\left\|f\left(x_{n}\right)-p\right\|^{2}+\left(1-\alpha_{n}\right)\left\|J_{\theta_{n}}^{B}\left(I-\theta_{n} A\right) u_{n}-J_{\theta_{n}}^{B}\left(I-\theta_{n} A\right) p\right\|^{2} \\
\leq \alpha_{n}\left\|f\left(x_{n}\right)-p\right\|^{2}+\left(1-\alpha_{n}\right)\left[\left\|u_{n}-p\right\|^{2}+a(b-2 \alpha)\left\|A u_{n}-A p\right\|^{2}\right] .
\end{gathered}
$$

Therefore, we have

$$
\begin{aligned}
\left(1-\alpha_{n}\right) a(2 \alpha-b)\left\|A u_{n}-A p\right\|^{2} \leq & \left\|x_{n}-p\right\|^{2} \\
& -\left\|x_{n+1}-p\right\|^{2}+\alpha_{n}\left\|f\left(x_{n}\right)-p\right\|^{2} .
\end{aligned}
$$

Since, $\alpha_{n} \rightarrow 0$ as $n \rightarrow \infty$, inequality (10) and $\left\{x_{n}\right\}$ is bounded, we obtain

$$
\lim _{n \rightarrow \infty}\left\|A u_{n}-A p\right\|^{2}=0 .
$$

Since $J_{\theta_{n}}^{B}$ is 1-inverse strongly monotone and (7), we have

$$
\begin{aligned}
& \left\|J_{\theta_{n}}^{B}\left(I-\theta_{n} A\right) u_{n}-p\right\|^{2} \\
\leq & \left\langle J_{\theta_{n}}^{B}\left(I-\theta_{n} A\right) u_{n}-J_{\theta_{n}}^{B}\left(I-\theta_{n} A\right) p,\left(I-\theta_{n} A\right) u_{n}-\left(I-\theta_{n} A\right) p\right\rangle \\
= & \frac{1}{2}\left[\left\|\left(I-\theta_{n} A\right) u_{n}-\left(I-\theta_{n} A\right) p\right\|^{2}+\left\|_{\theta_{n}}^{B}\left(I-\theta_{n} A\right) u_{n}-p\right\|^{2}\right. \\
& \left.-\left\|\left(I-\theta_{n} A\right) u_{n}-\left(I-\theta_{n} A\right) p-\left(J_{\theta_{n}}^{B}\left(I-\theta_{n} A\right) u_{n}-p\right)\right\|^{2}\right] \\
\leq & \frac{1}{2}\left[\left\|u_{n}-p\right\|^{2}+\left\|J_{\theta_{n}}^{B}\left(I-\theta_{n} A\right) u_{n}-p\right\|^{2}-\left\|u_{n}-J_{\theta_{n}}^{B}\left(I-\theta_{n} A\right) u_{n}\right\|^{2}\right. \\
& \left.+2 \theta_{n}\left\langle J_{\theta_{n}}^{B}\left(I-\theta_{n} A\right) u_{n}-p, A u_{n}-A p\right\rangle-\theta_{n}^{2}\left\|A u_{n}-A p\right\|^{2}\right] .
\end{aligned}
$$

So, we obtain

$$
\begin{aligned}
& \left\|J_{\theta_{n}}^{B}\left(I-\theta_{n} A\right) u_{n}-p\right\|^{2} \\
\leq & \left\|x_{n}-p\right\|^{2}-\left\|u_{n}-J_{\theta_{n}}^{B}\left(I-\theta_{n} A\right) u_{n}\right\|^{2} \\
& +2 \theta_{n}\left\langle J_{\theta_{n}}^{B}\left(I-\theta_{n} A\right) u_{n}-p, A u_{n}-A p\right\rangle-\theta_{n}{ }^{2}\left\|A u_{n}-A p\right\|^{2},
\end{aligned}
$$


and thus

$$
\begin{gathered}
\left\|x_{n+1}-p\right\|^{2}=\left\|\alpha_{n}\left(f\left(x_{n}\right)-p\right)+\left(1-\alpha_{n}\right)\left(J_{\theta_{n}}^{B}\left(I-\theta_{n} A\right) u_{n}-p\right)\right\|^{2} \\
\leq \alpha_{n}\left\|f\left(x_{n}\right)-p\right\|^{2}+\left(1-\alpha_{n}\right)\left\|J_{\theta_{n}}^{B}\left(I-\theta_{n} A\right) u_{n}-p\right\|^{2} \\
\leq \alpha_{n}\left\|f\left(x_{n}\right)-p\right\|^{2}+\left\|x_{n}-p\right\|^{2}-\left(1-\alpha_{n}\right)\left\|u_{n}-J_{\theta_{n}}^{B}\left(I-\theta_{n} A\right) u_{n}\right\|^{2} \\
-\left(1-\alpha_{n}\right) \theta_{n}{ }^{2}\left\|A u_{n}-A p\right\|^{2}+2\left(1-\alpha_{n}\right) \theta_{n}\left\langle J_{\theta_{n}}^{B}\left(I-\theta_{n} A\right) u_{n}-p, A u_{n}-A p\right\rangle .
\end{gathered}
$$

Since, $\alpha_{n} \rightarrow 0$ as $n \rightarrow \infty$, from inequalities (10) and (14), we obtain

$$
\lim _{n \rightarrow \infty}\left\|u_{n}-J_{\theta_{n}}^{B}\left(I-\theta_{n} A\right) u_{n}\right\|^{2}=0 .
$$

Next, we prove that $\limsup _{n \rightarrow+\infty}\left\langle p-f(p), p-x_{n}\right\rangle \leq 0$. Since $H$ is a Hilbert space and $\left\{x_{n}\right\}$ is bounded, there exists a subsequence $\left\{x_{n_{k}}\right\}$ of $\left\{x_{n}\right\}$ which converges weakly to $x^{*}$ in $K$ and

$$
\limsup _{n \rightarrow+\infty}\left\langle p-f(p), p-x_{n}\right\rangle=\lim _{k \rightarrow+\infty}\left\langle p-f(p), p-x_{n_{k}}\right\rangle
$$

Using (7) and Lemma 2.5, we arrive at

$$
\begin{aligned}
\left\|x_{n}-J_{\lambda}^{g} x_{n}\right\| & \leq\left\|u_{n}-J_{\lambda}^{g} x_{n}\right\|+\left\|u_{n}-x_{n}\right\| \\
& \leq\left\|J_{\lambda_{n}}^{g} x_{n}-J_{\lambda}^{g} x_{n}\right\|+\left\|u_{n}-x_{n}\right\| \\
& \leq\left\|u_{n}-x_{n}\right\|+\left\|J_{\lambda}^{g}\left(\frac{\lambda_{n}-\lambda}{\lambda_{n}} J_{\lambda_{n}}^{g} x_{n}+\frac{\lambda}{\lambda_{n}} x_{n}\right)-J_{\lambda}^{g} x_{n}\right\| \\
& \leq\left\|u_{n}-x_{n}\right\|+\left\|\frac{\lambda_{n}-\lambda}{\lambda_{n}} J_{\lambda_{n}}^{g} x_{n}+\frac{\lambda}{\lambda_{n}} x_{n}-x_{n}\right\| \\
& \leq\left\|u_{n}-x_{n}\right\|+\left(1-\frac{\lambda}{\lambda_{n}}\right)\left\|u_{n}-x_{n}\right\| \\
& \leq\left(2-\frac{\lambda}{\lambda_{n}}\right)\left\|u_{n}-x_{n}\right\| .
\end{aligned}
$$

Hence,

$$
\lim _{n \rightarrow \infty}\left\|x_{n}-J_{\lambda}^{g} x_{n}\right\|=0 .
$$

Since $J_{\lambda}^{g}$ is single valued and nonexpansive, using (16) and Lemma 2.1, then $x^{*} \in F i x\left(J_{\lambda}^{g}\right)=\operatorname{argmin}_{u \in K} g(u)$. Let us show $x^{*} \in(A+B)^{-1}(0)$. Since $A$ be an $\alpha$-inverse strongly monotone, $A$ is Lipschitz continuous monotone mapping. It follows from Lemma 2.7 that $B+A$ is maximal monotone. Let $(v, u) \in$ $G(B+A)$, i.e., $u-A v \in B(v)$. We set $z_{n}:=J_{\theta_{n}}^{B}\left(u_{n}-\theta_{n} A u_{n}\right)$. Since $z_{n_{k}}=$ 
$J_{\theta_{n_{k}}}^{B}\left(u_{n_{k}}-\theta_{n_{k}} A u_{n_{k}}\right)$, we have $u_{n_{k}}-\theta_{n_{k}} u_{n_{k}} \in\left(I+\theta_{n_{k}} B\right) z_{n_{k}}$, i.e., $\frac{1}{\theta_{n_{k}}}\left(u_{n_{k}}-\right.$ $\left.z_{n_{k}}-\theta_{n_{k}} A u_{n_{k}}\right) \in B\left(z_{n_{k}}\right)$. By maximal monotonicity of $B+A$, we have

$$
\left\langle v-z_{n_{k}}, u-A v-\frac{1}{\theta_{n_{k}}}\left(u_{n_{k}}-z_{n_{k}}-\theta_{n_{k}} A u_{n_{k}}\right)\right\rangle \geq 0
$$

and so

$$
\begin{aligned}
\left\langle v-z_{n_{k}}, u\right\rangle & \geq\left\langle v-z_{n_{k}}, A v-\frac{1}{\theta_{n_{k}}}\left(u_{n_{k}}-z_{n_{k}}-\theta_{n_{k}} A u_{n_{k}}\right)\right\rangle \\
& =\left\langle v-z_{n_{k}}, A v-A z_{n_{k}}+A z_{n_{k}}+\frac{1}{\theta_{n_{k}}}\left(u_{n_{k}}-z_{n_{k}}-\theta_{n_{k}} A u_{n_{k}}\right)\right\rangle \\
& \geq\left\langle v-z_{n_{k}}, A z_{n_{k}}-A u_{n_{k}}\right\rangle+\left\langle v-z_{n_{k}}, \frac{1}{\theta_{n_{k}}}\left(u_{n_{k}}-z_{n_{k}}\right)\right\rangle .
\end{aligned}
$$

It follows from $\left\|z_{n}-u_{n}\right\| \rightarrow 0,\left\|A z_{n}-A u_{n}\right\| \rightarrow 0$ and $z_{n_{k}}$ converges weakly to $x^{*}$, we get

$$
\lim _{k \rightarrow+\infty}\left\langle v-z_{n_{k}}, u\right\rangle=\left\langle v-x^{*}, u\right\rangle \geq 0
$$

and hence $x^{*} \in(A+B)^{-1}(0)$. Therefore, $x^{*} \in(A+B)^{-1}(0) \cap \operatorname{argmin}_{u \in K} g(u)$. On other hand, the fact that $p$ solves (8), we then have

$$
\begin{aligned}
\limsup _{n \rightarrow+\infty}\left\langle p-f(p), p-x_{n}\right\rangle & =\lim _{k \rightarrow+\infty}\left\langle p-f(p), p-x_{n_{k}}\right\rangle \\
& =\left\langle p-f(p), p-x^{*}\right\rangle \leq 0 .
\end{aligned}
$$

Finally, we show that $x_{n} \rightarrow p$. From (7) and Lemma 2.3, we get that

$$
\begin{aligned}
\left\|x_{n+1}-p\right\|^{2}= & \left\|\alpha_{n} f\left(x_{n}\right)+\left(1-\alpha_{n}\right) J_{\theta_{n}}^{B}\left(I-\theta_{n} A\right) u_{n}-p\right\|^{2} \\
\leq & \left\|\alpha_{n}\left(f\left(x_{n}\right)-f(p)\right)+\left(1-\alpha_{n}\right)\left(J_{\theta_{n}}^{B}\left(I-\theta_{n} A\right) u_{n}-p\right)\right\|^{2} \\
& +2 \alpha_{n}\left\langle p-f(p), p-x_{n+1}\right\rangle \\
\leq & \left(\alpha_{n}\left\|f\left(x_{n}\right)-f(p)\right\|+\left\|\left(1-\alpha_{n}\right)\left(J_{\theta_{n}}^{B}\left(I-\theta_{n} A\right) u_{n}-p\right)\right\|\right)^{2} \\
& +2 \alpha_{n}\left\langle p-f(p), p-x_{n+1}\right\rangle \\
\leq & \left(\alpha_{n} b\left\|x_{n}-p\right\|+\left(1-\alpha_{n}\right)\left\|J_{\theta_{n}}^{B}\left(I-\theta_{n} A\right) u_{n}-p\right\|\right)^{2} \\
& +2 \alpha_{n}\left\langle p-f(p), p-x_{n+1}\right\rangle \\
\leq & \left(\left(1-\alpha_{n}(1-b)\right)\left\|x_{n}-p\right\|\right)^{2}+2 \alpha_{n}\left\langle p-f(p), p-x_{n+1}\right\rangle \\
\leq & \left(1-\alpha_{n}(1-b)\right)\left\|x_{n}-p\right\|^{2}+2 \alpha_{n}\left\langle p-f(p), p-x_{n+1}\right\rangle .
\end{aligned}
$$


The conclusion that $x_{n} \rightarrow p$. follows from Lemma 2.4.

Case 2. The sequence $\left\{\left\|x_{n}-p\right\|\right\}$ is not eventually decreasing. Set $\Pi_{n}=\| x_{n}-$ $p \|^{2}$ and $\tau: \mathbb{N} \rightarrow \mathbb{N}$ be a mapping for all $n \geq n_{0}$ (for some $n_{0}$ large enough) by $\tau(n)=\max \left\{k \in \mathbb{N}: k \leq n, \Pi_{k} \leq \Pi_{k+1}\right\}$. We have $\tau$ is a non-decreasing sequence such that $\tau(n) \rightarrow \infty$ as $n \rightarrow \infty$ and $\Pi_{\tau(n)} \leq \Pi_{\tau(n)+1}$ for $n \geq n_{0}$. By following the same line of arguments as in Case 1, we can show that $\left\{x_{\tau(n)}\right\}_{n \geq 1}$ and $\left\{y_{\tau(n)}\right\}_{n \geq 1}$ are bounded in $K$ and $\left.\limsup \left\langle p-f(p), p-x_{\tau(n)}\right)\right\rangle \leq 0$. We have for all $n \geq n_{0}$,

$$
\begin{aligned}
0 & \leq\left\|x_{\tau(n)+1}-p\right\|^{2}-\left\|x_{\tau(n)}-p\right\|^{2} \\
& \leq \alpha_{\tau(n)}\left[-(1-b)\left\|x_{\tau(n)}-p\right\|^{2}+2\left\langle p-f(p), p-x_{\tau(n)+1}\right\rangle\right],
\end{aligned}
$$

which implies that

$$
\left\|x_{\tau(n)}-p\right\|^{2} \leq \frac{2}{1-b}\left\langle p-f(p), p-x_{\tau(n)+1}\right\rangle .
$$

Then, we have

$$
\lim _{n \rightarrow \infty}\left\|x_{\tau(n)}-p\right\|^{2}=0
$$

Therefore,

$$
\lim _{n \rightarrow \infty} \Pi_{\tau(n)}=\lim _{n \rightarrow \infty} \Pi_{\tau(n)+1}=0 .
$$

Furthermore, for all $n \geq n_{0}$, we have $\Pi_{\tau(n)} \leq \Pi_{\tau(n)+1}$ if $n \neq \tau(n)$ (that is, $n>\tau(n)$ ); because $\Pi_{j}>\Psi_{j+1}$ for $\tau(n)+1 \leq j \leq n$. As consequence, we have for all $n \geq n_{0}$,

$$
0 \leq \Pi_{n} \leq \max \left\{\Pi_{\tau(n)}, \Pi_{\tau(n)+1}\right\}=\Pi_{\tau(n)+1} .
$$

Hence, $\lim _{n \rightarrow \infty} \Pi_{n}=0$, that is $\left\{x_{n}\right\}$ converges strongly to $p$. This completes the proof.

Now, we give some remarks on our results as follows:

(1) The proof methods of our result are very different from Boikanyo's method [2] for monotone inclusion problem. Further, we remove the following conditions:

(i) $\liminf r_{n}>0$,

(ii) $0<\liminf \delta_{n}<\lim \sup \delta_{n}<\frac{3}{2}$,

(iii) $\left\|e_{n}\right\| \leq \gamma_{n}\left\|x_{n}-\delta_{n} J_{r_{n}}^{B}\left(x_{n}-r_{n} A x_{n}\right)\right\|$ with $\sum_{n=0}^{\infty} \gamma_{n}{ }^{2}<\infty$ in [2]. 
(2) Our results improve many recent results using proximal point algorithms for solving variational inclusion problem (3) and convex minimization problem (1) in real Hilbert spaces.

(3) Our results are applicable for finding a common solution of some nonlinear problem, namely, equilibrium problem, composite optimization problem, inclusion problem.

\section{Bibliography}

[1] L. Ambrosio, N. Gigli and G. Savaré, Gradient Flows in Metric Spaces and in the Space of Probability Measures, Second edition, Lectures in Mathematics ETH Zürich, Birkhäuser Verlag, Basel, 2008.

[2] O. A. Boikanyo, The viscosity approximation forward-backward splitting method for zeros of the sum of monotone operators, Abstract and Applied Analysis 2016 (2016), 1-10.

[3] S. Chang, Y. Tang, L. Wang and G. Wang, Convergence theorems for some multivalued generalized nonexpansive mappings, Fixed Point Theory and Applications 2014 (2014), Article number: 33.

[4] G. H-G. Chen and R. T. Rockafellar, Convergence rates in forward-backward splitting, SIAM J. Optim. 7(2) (1997), 421-444.

[5] C. E. Chidume, Geometric Properties of Banach spaces and Nonlinear Iterations, vol. 1965 of Lecture Notes in Mathematics, Springer-Verlag, 2009.

[6] J. Jost, Convex functionals and generalized harmonic maps into spaces of nonpositive curvature, Comment. Math. Helv. 70 (1995), 659-673.

[7] L. Genaro, M. M. Victoria, W. Fenghui and H. K. Xu, Forward-backward splitting methods for accretive operators in Banach spaces, Abstract and Applied Analysis 2012 (2012), Article ID 109236, 25 pages.

[8] O. Güler, On the convergence of the proximal point algorithm for convex minimization, SIAM J. Control Optim. 29 (1991), 403-419.

[9] S. Kamimura and W. Takahashi, Approximating solutions of maximal monotone operators in Hilbert spaces, J. Approx. Theory 106 (2000), 226-240.

[10] S. Kamimura and W. Takahashi, Strong convergence of a proximal-type algorithm in a Banach space, SIAM J. of Optimization 13(3) (2003), 938-945.

[11] N. Lehdili and A. Moudafi, Combining the proximal algorithm and Tikhonov regularization, Optimization 37 (1996), 239-252.

[12] P. L. Lions and B. Mercier, Splitting algorithms for the sum of two nonlinear operators, SIAM J. Numer. Anal. 16 (1979), 964-979. 
[13] P. E. Mainge, Strong convergence of projected subgradient methods for nonsmooth and nonstrictly convex minimization, Set-Valued Analysis 16 (2008), 899-912.

[14] B. Martinet, Régularisation d'inéquations variationnelles par approximations successives, Rev. Franaise Informat. Recherche Opérationnelle 4 (1970), 154-158 (in French).

[15] I. Miyadera, Nonlinear Semigroups, Translations of Mathematical Monographs, American Mathematical Society, 1992.

[16] G. B. Passty, Ergodic convergence to a zero of the sum of monotone operators in Hilbert spaces, J. Math. Anal. Appl. 72, 383-390 (1979).

[17] D. H. Peaceman and H. H. Rachford, The numerical solution of parabolic and elliptic differential equations, J. Soc. Indust. Appl. Math. 3(1) (1955), 28-41.

[18] R. T. Rockafellar, Monotone operators and the proximal point algorithm, SIAM J. Control Optimization 14 (1976), 877-898.

[19] S. Reich, Strong convergence theorems for resolvents of accretive operators in Banach spaces, J. Math. Anal. Appl. 183 (1994), 118-120.

[20] T. M. M. Sow, An iterative algorithm for solving equilibrium problems, variational inequality problems and fixed point problems with multivalued quasi-nonexpansive mappings, Appl. Set-Valued Anal. Optim. 1(2) (2019), 171-185.

[21] M. V. Solodov and B. F. Svaiter, Forcing strong convergence of proximal point iterations in a Hilbert space, Math. Program., Ser. A 87 (2000), 189-202.

[22] P. Tseng, A modified forward-backward splitting method for maximal monotone mappings, SIAM J. Control Optim. 38(2) (2000), 431-446.

[23] H. K. Xu, Inequalities in Banach spaces with applications, Nonlinear Anal. 16(12) (1991), 1127-1138.

[24] H. K. Xu, A regularization method for the proximal point algorithm, J. Glob. Optim. 36 (2006), 115-125.

Received November 30, 2019; revised April 14, 2020; accepted June 10, 2020.

\section{Author information}

Thierno M. M. Sow, Amadou Mahtar Mbow University, Dakar, Senegal.

E-mail: sowthierno89@gmail.com 